(C) 2021 IEEE. Personal use of this material is permitted. Permission from IEEE must be obtained for all other uses, in any current or future media, including reprinting/republishing this material for advertising or promotional purposes, creating new collective works, for resale or redistribution to servers or lists, or reuse of any copyrighted component of this work in other works

The final published version can be found at https://doi.org/10.1109/JSAC.2020.3042421 


\title{
Internet of Things for In-Home Health Monitoring Systems: Current Advances, Challenges and Future Directions
}

\author{
Nada Y. Philip, Senior Member, IEEE, Joel J. P. C. Rodrigues, Fellow, IEEE, \\ Honggang Wang, Senior Member, IEEE, Simon Fong, Jia Chen
}

\begin{abstract}
Internet of Things has been one of the catalysts in revolutionizing conventional healthcare services. With the growing society, traditional healthcare systems reach their capacity in providing sufficient and high-quality services. The world is facing the aging population and the inherent need for assisted-living environments for senior citizens. There is also a commitment by national healthcare organizations to increase support for personalized, integrated care to prevent and manage chronic conditions. Many applications related to In-Home Health Monitoring have been introduced over the last few decades, thanks to the advances in mobile and Internet of Things technologies and services. Such advances include improvements in optimized network architecture, indoor networks coverage, increased device reliability and performance, ultra-low device cost, low device power consumption, and improved device and network security and privacy. Current studies of in-home health monitoring systems presented many benefits including improved safety, quality of life and reduction in hospitalization and cost. However, many challenges of such a paradigm shift still exist, that need to be addressed to support scale-up and wide uptake of such systems, including technology acceptance and adoption by patients, healthcare providers and policymakers. The aim of this paper is three folds: First, review of key factors that drove the adoption and growth of the IoT-based in-home remote monitoring; Second, present the latest advances of IoT based in-home remote monitoring system architecture and key building blocks; Third, discuss future outlook and our recommendations of the in-home remote monitoring applications going forward.
\end{abstract}

Index Terms - Internet of Things, IoT, Ambient assisted living, eHealth, In-home, mHealth, Remote monitoring, Middleware, Tutorial.

\section{INTRODUCTION}

$\mathrm{I}^{\mathrm{N}}$ N-HOME health monitoring allows patient care to continue at home after a patient is discharged from the hospital. It

This work was supported in part by FCT/MCTES through national funds and when applicable co-funded EU funds under the project UIDB/50008/2020, in part by the Brazilian National Council for Scientific and Technological Development (CNPq) via Grant No. 309335/2017-5.

Nada Y. Philip is with the Faculty of Science, Engineering and Mathematics at Kingston University London, UK (n.philip@kingston.ac.uk).

Joel J. P. C. Rodrigues is with the Federal University of Piauí (UFPI), Teresina-PI, Brazil and with Instituto de Telecomunicações, Portugal (e-mail: joeljr@ieee.org). allows healthcare providers to reach patients outside of the four walls of the hospital, perform proper monitoring of patient health conditions, continue to deliver quality care and identify at-risk populations. It also helps patients stay connected with their health providers, enable them to remain compliant with treatment plans and improve their health conditions.

Internet of Things (IoT) based in-home health monitoring applications are one of the key mobile health (mHealth) applications that provide proactive and preventive digital health interventions [1],[2]. Digital health is "a broad umbrella term encompassing eHealth (which includes mHealth), as well as emerging areas, such as the use of advanced computing sciences in 'big data', genomics and artificial intelligence"[2]. Whereas mHealth can be defined as "mobile computing, medical sensor, and communications technologies for health care" [3].

Over the years there has been a booming in the number of mHealth applications in the market. According to Global Market Insights, mHealth Market size is set to exceed \$289.4 billion by 2025 [5].

This prolific increase in mHealth applications and in-home health monitoring is due to three main factors:

\section{A. Current healthcare services limitations and health policymakers' planning directions.}

Globally, the population aged 65 and over is growing faster than all other age groups. According to an estimate by the World Health Organization (WHO), the number of individuals over 60 years will nearly double from $12 \%$ to $22 \%$ during the 2015-2050 period. Approximately $80 \%$ of older adults have at least one chronic disease and $77 \%$ have at least two [4].

The incoming 'Silver Tsunami' will generate higher medical needs and caregiving, which will ultimately place more and

Honggang Wang is with University of Massachusetts Dartmouth, North Dartmouth, MA, USA (emai:hwang1@umassd.edu)

Simon Fong is with the Faculty of Science and Technology, University of Macau, Macau SAR and with ZIAT of Chinese Academy of Science, China (ccfong@um.edu.mo).

Jia Chen is with IBM (jia.chen.nyc@gmail.com). 
more pressure on an already stressed healthcare system. Therefore, finding novel ways and leveraging technology to manage the health of populations efficiently and cost effectively, will be the key to sustainably provide the best quality of care. Accordingly, treating individuals remotely with the support of technology is on the agenda and plan of national and global health policymakers. Recently the WHO organization created a framework for categorizing digital health interventions [2]:

- Interventions for clients: clients are individuals who are potential or current users of health services.

- Interventions for healthcare providers: Healthcare providers are members of the health workforce who deliver health services.

- Interventions for a health system or resource managers: managers are involved in the administration and planning of public health systems.

- Interventions for data services: This includes functionality to support steps related to data collection, management and processing.

In most of the above intervention groupings, remote health monitoring applications are part of the end-to-end intervention system. Remote health monitoring applications links the client via sensors and home hub to the healthcare providers and resource managers via cloud data services.

To address limited health and social care resources, National Health Services' (NHS) policies and plans are set to change the services models to keep patients treatment at home and community centers to reduce hospitalization, cost and provide a better quality of life. As an example, NHS UK, sets out five changes to the NHS service model to address such needs [6]:

- Boost 'out-of-hospital' care: to dissolve the historic divide between primary and community health services.

- NHS redesign: to reduce pressure on emergency services at hospitals.

- Empowering patients and personalized care: patients to get more control over their health.

- Digitally-enabled care: to be part of the primary and outpatient care pathways across the NHS.

- Integrated care systems: to focus on population health and partnerships between local NHS organizations.

The key enabler of these 5 major practical changes to the health service model is the remote health monitoring applications. The above mentioned 'out of hospital' care, reducing emergency hospital services, personalized care, digitally enables care and integrated care systems models cannot happen without remote health monitoring types of applications. In fact, the fast increasing focus towards precision medicine and personalized care is one of the factors fueling the global mHealth market [5].

B. The advances in the underlying enabling technologies in terms of mobile phone capabilities, wireless communications, sensors, wearables and IoT architectures and protocols.

The relatively low cost and proliferation of mHealth applications, due to the massive penetration of smartphones, is making it a promising investment direction across the globe. In 2017, it is estimated that 500 million smartphones new users from China and India were connected to the internet globally [5].

Internet of Things (IoT) is an evolving IT revolution providing a paradigm shift in several areas including Healthcare. The term "Internet-of-Things" can be defined as " an umbrella keyword to cover various aspects related to the extension of the Internet and the Web into the physical realm, by means of the widespread deployment of spatially distributed devices with embedded identification, sensing and/or actuation capabilities, to enable a whole new class of applications and services" [7]. Such applications will continue to rise due to the development of the recent communication protocol specifically designed for IoT devices such as NB-IoT, LoraWan or Sigfox. In addition, the latest development in the IoT communication infrastructure including 3GPP standard (5G IoT) is well positioned to provide low-power, low-data-rate, and wide-area coverage cellular connections to diverse types of IoT devices [8].

There has been an increase in the development of intelligent medical devices (e.g. blood pressure device, glucose meter, temperature sensors, weight scale, etc..) and wearable sensors (to measure e.g. ECG, accelerometer, SPo2, Heart rate, etc..), with features focusing on low power, small size, portability and easy to wear and use. Wearable sensors have gradually been developed in the form of accessories (e.g. bands, rings), smart clothing, body attachments and body insertions (e.g. insulin pumps, pacemakers). Alongside this development in wearable sensors, there have been advancements in the design of smart textiles, smart clothing, or e-textile, that consist of conductive textile material that is attached to or woven together. The tremendous advancements in low-profile and bioelectronics, nano technologies and materials have led to the development of implantable sensors and biomedical devices for remote diagnosis and monitoring. Many challenges were resolved during this development including the size of the sensors, battery life and the development of stretchable and skinattachable electronic devices that can continuously and unobtrusively monitor individuals' activity and biomedical signals without any restriction to the individual's daily activities. Wearable devices are equipped with wireless transceivers modalities, e.g. Bluetooth, Zigbee, infrared, radiofrequency identification (RFID), WiFi and near-field communication (NFC) technologies. Such technologies allow the wearables to connect to other smart devices (e.g. Smartphone) to enable remote diagnosis and monitoring for better quality care [9].

\section{$C$. The reported evidence on the benefits of mHealth applications in terms of quality of care and reduction of cost}

In-Home health monitoring applications have evolved over the last few decades, addressing many healthcare conditions. They aimed to provide more efficient and effective healthcare services and contributed to a better quality of life and reduction in cost. There has been a sharp increase in the number of mHealth smartphone applications targeting various disease 
remote monitoring and self-management, helping patients better manage their health conditions and enabling independent living. It aims to empower individuals through disease prevention, health promotion and condition self-management [5].

Based on [12] an estimated 7.1 million patients were remotely connected to health monitoring devices in 2016, which contributed to a saving of $£ 1$ bn (over five years) for the NHS by reducing bed blocking and unnecessary appointments.

In addition to the above mentioned benefits of remote health monitoring, it gives patients confidence that their conditions (e.g. heart rate, blood pressure, SPo2 levels and sleep quality) are monitored and alerts could be generated to inform their healthcare professionals in real time [12]. In fact, one study has shown that home monitoring of patients with congestive heart failure leads to lower hospitalization rates and improved mortality [14]. A meta-analysis study on the effectiveness of mHealth interventions for patients with diabetes reported that over a period of a year on average, mHealth interventions improve glycemic control (HbAlc) compared to conventional care by as much as $0.8 \%$ for patients with type 2 diabetes and $0.3 \%$ for patients with type 1 diabetes [11].

In this paper, we first set the stage by presenting the main components of IoT based In-home health monitoring system along with some examples. Inspired by the main technology building blocks of IoT based in-home health monitoring systems, section II provides an extensive discussion on the technology advances used in such systems. Section III identifies the main challenges and future directions in developing successful IoT in-home health monitoring systems that could scale up and lead to successful deployment in national healthcare services. Section IV concludes the paper with a summary.

\section{CuRrent AdVANCES IN IOT TEChNOLOGIES AND SERVICES FOR IN-HOME HEALTH MONITORING APPLICATIONS}

IoT technology is one of the main enablers of in-home health monitoring system architecture. Fig. 1 represents a typical example of the main building blocks of IoT in-home health monitoring systems. Fig. 1 demonstrates the functional modules of such systems and their interaction. The cloud hub of the system consists of several modules (storage server, feature extraction module and decision support system). The patient hub handles the interaction with the patient, wearable sensors and devices and the transfer of the patient's vital signs and the receiver of the treatment plan. Healthcare professional hub applications interact with the medical staff and facilitate patients' treatment. In IoT based in-home health monitoring systems, the communication between the cloud hub and other user applications related to the patient's and healthcare professional hub is via interoperable and secure Cloud Communications API (e.g. based on RESTful web services).

There has been a tremendous increase of such applications for chronic disease self-management (e.g. Diabetes and Cancer), medication adherence (Smart pills), assistive living (Parkinson and mild cognitive conditions) and many more.
Table I present a summary of several of such applications. It includes a brief description of the systems, their advantages and lists the sensors used in the remote monitoring [53] - [62].

The architecture of IoT based in-home health monitoring systems typically includes five main key IoT technologies as shown in Fig. 2. Inspired by these five technologies, the following sub-sections presents the current advances in IoT technologies and services for in-home health monitoring applications.

\section{A. mHealth and assistive sensors}

These represent invasive and non-invasive sensors used to monitor biomedical signals and living environment changes. Biomedical signals depend on the individual's lifestyle, mental and medical conditions (e.g. diabetes, COPD, Cancer and mental disorder). Such medical conditions need the management and control of some parameters, e.g. Glucose level, blood pressure, temperature, ECG and weight. And hence the need for sensors devices to measure these conditions. For the living environment, it depends on the assistive living technologies that individuals needs, e.g. personal alarms, sensor mats, camera, etc. To enable communications of the measured signal with the surrounding world, these sensors are attached to wireless communications modalities including, RFID, NFC, Bluetooth and BLE, WiFi and Zigbee.

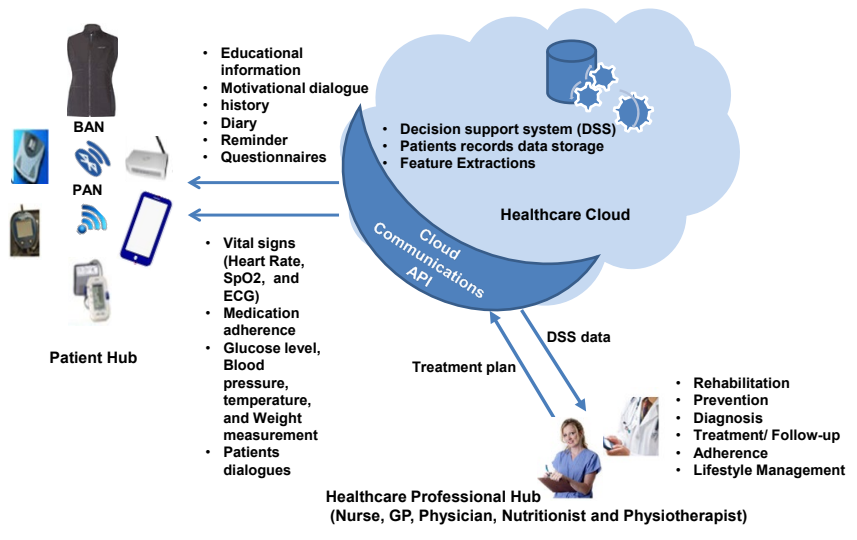

Fig. 1. IoT based in-home remote monitoring system architecture

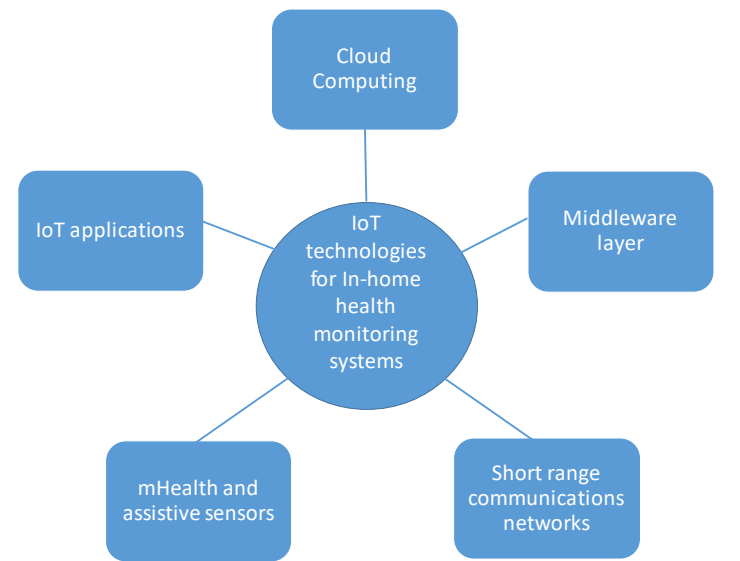

Fig. 2. Key technologies for IoT based In-home health monitoring systems 
TABLE I

EXAMPLES OF MHEALTH AND ASSISTED LIVING SENSORS AND APPLICATIONS

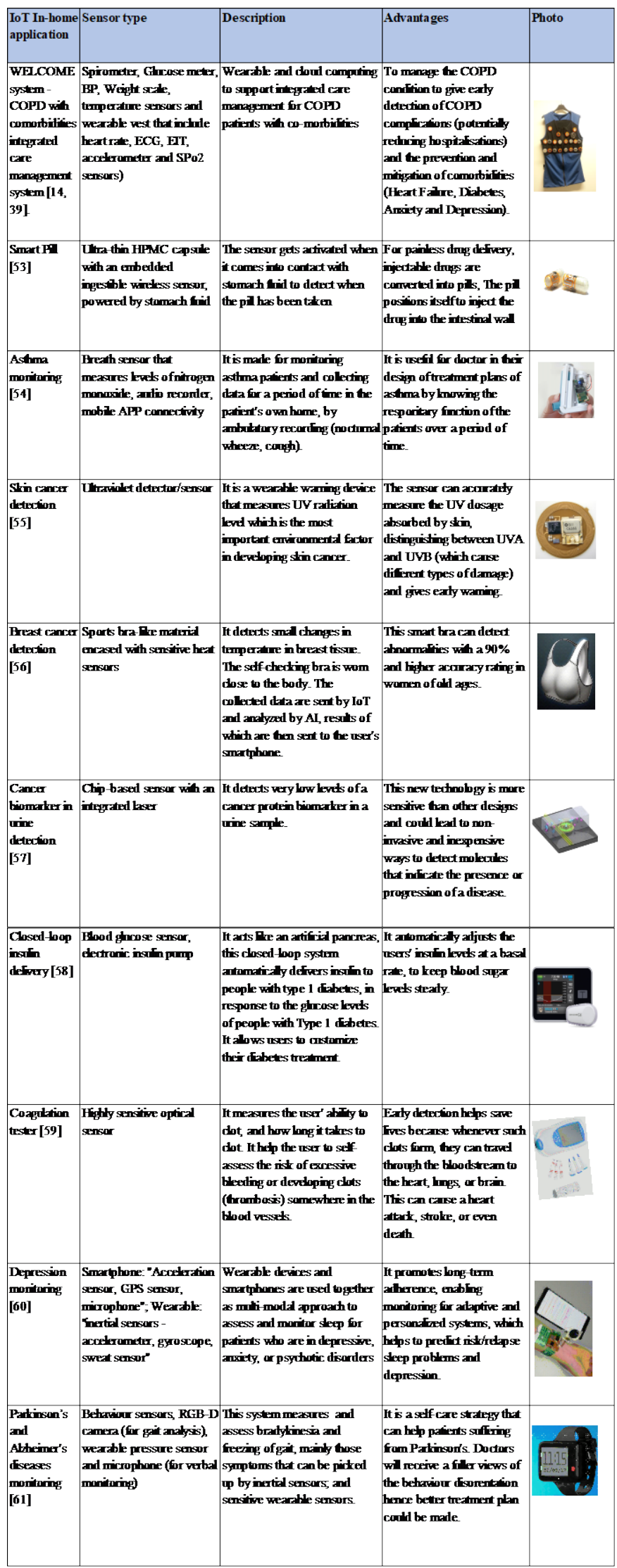

Table 1 represents a list of sensors and devices related to some chronic disease management, adherence and assisted living applications. Most of these sensors and devices are manufactured with data communications standards to allow interoperability and communications with applications. Examples of such standards are the ISO/IEEE 11073 Personal Health Data (PHD) and oneM2M. They both are designed to allow message communications with devices using application layer protocols including Hyper TextTransfer Protocol (HTTP), Constrained Application Protocol (CoAP), and Message Queuing Telemetry Transport (MQTT).

\section{B. Short-range communications networks}

Short-range communications networks are represented in this discussion as Wireless sensor networks (WSN) and Personal Area Networks (PAN): WSN, is a network composed of a set of sensors to monitor different health conditions and/or assistive living parameters. Usually, this is referred to as Wireless Body Area Network (WBAN) in the case of wearable required to be worn by individuals. PAN, is a network that allows communications between sensor(s) and personal computer devices, e.g. smartphone using short-range communications that include e.g. Bluetooth, BLE, WIFI and Zigbee. It depends on the required bandwidth. For instance, for sensor signals with a low bandwidth of $0.5 \mathrm{~Hz}$, such as $\mathrm{SPo} 2$ signal, BLE is sufficient to be used to transmit the data [16]. While 25 lead ECG signals bandwidth can reach $500 \mathrm{~Hz}$ would need WiFi communications modalities as the required bandwidth is high [16]. Fig. 3 presents some of the IoT communications protocols in terms of data rate and range [17].

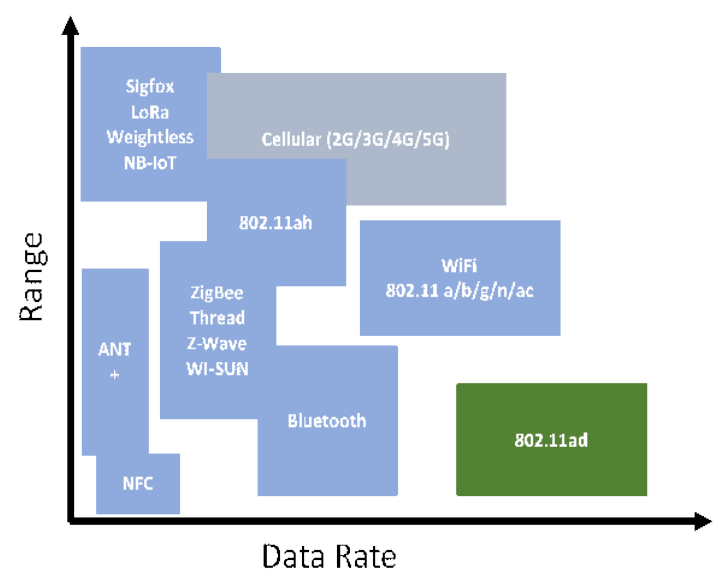

Fig. 3. IoT protocols in terms of range and data rate

Recent protocols are specifically designed for IoT devices such as NB-IoT, LoraWan or Sigfox. They are designed to use lowpower wide-area networks (LPWAN) that enable the connection of a large number of devices at a low bit rate, low energy consumption and low cost. In particular, the IEEE 802.15.6, is a wireless body area network (WBAN) standard developed for enhanced health monitoring, which supports data rates up to $10 \mathrm{Mbps}, 1-2$ meters range, low power, and high 
reliability [19][19]. The latest development in the IoT communication infrastructure includes the latest 3GPP standard development of (5G IoT) to provide low-power, low-data-rate, and wide-area coverage cellular connections to diverse types of IoT devices [8]. This includes two connection types $\{$ direct $3 \mathrm{GPP}$ connection via narrowband IoT (NB-IoT) and indirect non-3GPP connection. For NB-IoT, it requires merely $180 \mathrm{kHz}$ as the minimum bandwidth for both uplink and downlink. To support non-3GPP 5G IoT connections, a heterogeneous link combining a low-power wide-area network (LPWAN) and cellular networks via relay user equipment (UE) appears to be a promising option [8].

\section{Middleware Layer}

It is a service-oriented software layer that allows the communications with heterogeneous devices like sensors and actuators from one end and the cloud services at the other end. The central role of the middleware layer is to create an abstraction of underlying sensors. The middleware layer acts as a mediator between the cloud and sensor devices by collecting the sensed data and uploading them to the cloud platform. The middleware layer is usually developed in a unified way that allows communications with any type of sensor and runs any applications with minimum configurations [20]. A middleware layer provides interoperability among interconnected devices and offers an abstraction layer to facilitate the development of applications [21]. In other words, middleware is a software abstraction between the application layer and the system infrastructure. Middleware platforms enable physical mashups by creating and offering value-added services to applications based on the combination of available system resources [21]. The key requirements for a middleware layer are:

- It should support interoperability to support multiple heterogeneous devices.

- It should provide a high-level API to access the services that abstract the underlying devices.

Existing literature contains a plethora of research efforts offering middleware solutions for IoT applications with varied scopes [23]. The main goal of most of the existing middleware systems and research projects was to build a unified adaptation layer framework to support the important needs of the respective domains. Some of these efforts focus on providing integration to existing middleware. For example, Mosden [24] is a middleware application to collect sensor data from external and mobile phone's internal sensors and uploads it to the GSN (Global Sensor Network) middleware.

UBIWARE [25], proposes an agent-based middleware to classify and register different devices as resources and then link them as components of business processes. It is built on top of the Java Agent Development Framework JADE. It represents each resource as a software agent (a Java component) that monitors the resource and supports the interoperation with other system elements. Each agent has a behavioural model to define the agent's role, which is represented in a UBIWARE specific rule-based semantic language called Semantic Agent Programming Language (SAPL). UBIWARE middleware is focused more on systems that can support multiple agents to control resources.

HYDRA [26]], which evolved into Linksmart, is a serviceoriented paradigm-based middleware. It is resource-aware, so it only hosts its Network Manager component on more powerful devices which are called Hydra-enabled devices. The restricted less powerful devices are connected to the Hydra network by a proxy that provides web service interfaces to access a device. Each Hydra-enabled device enables communication among devices and may host a proxy to act as a gateway. Many EU projects including, REACTION [27], inCASA [28], ebbits [29], MASSIF [30], SEEMPubS [31], SEAM4US [32], have employed HYDRA middleware. However, the interoperability of HYDRA is limited to Health Device Profile (HDP) devices [33]. Furthermore, HYDRA doesn't follow REST design principles.

MyHealthAssistant [49] is an Event-driven smartphone based Middleware architecture. It is efficiently designed to consume low energy and resources while collecting and merging data from BAN sensors and other assistive sensor networks.

OpenIoT middleware [34], an EU FP7 project, is focused on enabling IoT application development based on the utility cloud computing delivery model. It follows REST design principles and offers resources as RESTful web services. It also uses W3C Semantic Sensor Networks (SSN) for the description of sensors and utilizes IETF CoAP (Constrained Application Protocol) for machine to machine (M2M) communications. A comprehensive review of middleware platforms can be found in [19] [50][51] [65].

Driven by the Web-of-Things (WoT) vision the design for a typical patient hub platform architecture can be divided into three layers, as shown in Fig. 4: application layer, middleware layer and sensors layer. The application layer uses the middleware layer to gather the data from the sensor layer and send to the cloud. The middleware layer proposed in this architecture is based on RESTful web services that enable the WoT paradigm at the cloud to cope with the interoperability challenge by offering a standard interface. This paradigm treats all the available services as resources and provides Uniform Resource Identifiers (URIs) to interact with those resources using HTTPs, which allow the usage of widely adopted and tested standards. Based on the discussion above, the following are the main design goals of any middleware layer for IoT based in-home health remote monitoring applications:

- Heterogeneity: The design should be extensible to support heterogeneous wireless protocols, sensor devices and BAN.

- Mobility: The patient-hub application of in-home health remote monitoring system must run unobtrusively to monitor different health-related attributes while supporting the patient's mobility at home (e.g. engaging in some simple home activities like gardening). 
- Interoperability: It must be fully interoperable with the cloud, and must synchronize the recorded medical data to the cloud.

- Adaptability: The architecture should be modular and extensible to handle different sensor configuration changes.

- User Experience: Reliability and timeliness are the most important performance requirements of the patient-hub side of in-home health remote monitoring applications. This in addition to measures to meet high accessibility and usability requirements of the patienthub.

- Security: As typical requirements in any e-health platform, considerable security and privacy are required to support confidentiality, integrity and availability.

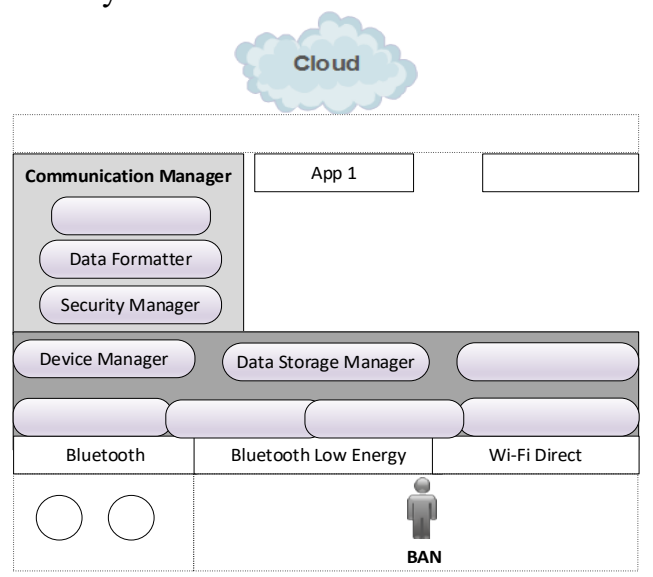

Fig. 4. Middleware software architecture design.

\section{Cloud computing}

Cloud computing is another sub-system of in-home health remote monitoring system. It is an internet-based computing platform that is utilized to provide; data storage to store the collected data from the different IoT devices and sensors, servers to process and analyse the collected data, and intelligent systems that used the analysed data to produce alarms and knowledge to support the decision of e.g. healthcare professional for patient treatment. Recently the concept of fog computing was introduced to provide a mix of local and cloudcomputing services that act as a highly virtualized platform that provides computing, storage, and networking services between end devices and traditional cloud computing data centers [63]. Many IoT cloud computing platforms were introduced that can be used as Infrastructure as a Service (IaaS), Platform as a service (PaaS) or Software as a Service (SaaS), Table II presents the most common IoT platforms and their key characteristics. The table lists these platforms along with their characteristics: connectivity, security, data format support, programming language support and analytics capabilities [35]. When selecting a cloud computing platform other factors are important to be considered like pricing model, availability, deployment type and hardware required.
Fig. 1 gives an example of cloud computing services required for an IoT based in-home remote management. These services can be hosted on one of the platforms in Table II platforms, e.g. Amazon Web Services. These cloud services consist of three main parts, the storage server, the feature extraction module and the decision support system (DSS). These modules handle the medical data provided by the end users. Typically, the cloud computing services are designed with three main objectives: flexibility, scalability and interoperability [15]. Interoperability is facilitated through the adoption of electronic health record handling standards e.g. HL7 FHIR to a seamless integration between systems. Scalability and flexibility are facilitated by the fact that new resources can easily be added to the model without changing the cloud communication API endpoints. The storage server saves the patient data, bio-signals and bioparameters transferred from the patient hub and medically relevant data resulted from the patient's evaluation by medical staff. The feature extraction module retrieves from the storage the raw signals originating from the patient hub (e.g. ECG). The module extracts several higher-level features to be used in the decision process. The decision support system (DSS) is designed based on semantic web technologies (e.g. RDF, OWL) and dynamic rules to enhance the inference process. Some typical functions provided by the decision support procedure include raising alarm, sending alerts, is to produce alarms, warnings and notifications about the status of patients' health based on the patients' extracted features.

\section{E. IoT applications}

IoT applications constitute the interface between the user and the devices. They enable device to device, humans to device, and human to human interactions.. They should be able to present data intuitively, identify problems and suggest solutions. Many research studies systematically reviewed the features of such applications for IoT based in-home health remote monitoring for chronic disease self-management. For example, the authors of [52] systematically reviewed the literature and identified the features for mobile diabetes applications while the authors of [33] systematically reviewed the literature identified the features of COPD self-management applications. Both studies were based on identifying the main features of IoT user applications. The following are examples of such features for chronic disease self-management applications:

- Measurement: This is device to device interaction; it allows communication between the application running on a smartphone and the sensors. In case of diabetes, measurements of blood glucose level.

- Questionnaires: This is a device to human interaction; it allows the collection of subjective status (e.g. mood) of the patients via dialogue or validated questionnaires.

- Educational: This is a device to human interaction; to provide personalized education based on patients' needs (e.g. nutrition information education). 
- History: This is a device to human interaction; to provide details about the collected data over a period of time.

- Diary and reminder: This is a device to human interaction; to remind patients about the treatment plan schedule.

- Communications: This is human to human electronic communications. E.g. via motivational messaging between Healthcare professional and patients.

- Medication: this is device to human interaction; it provides e.g. the list of medications, frequency and dosage.

TABLE II

IOT CLOUD COMPUTING INDUSTRIAL PLATFORMS

\begin{tabular}{|c|c|c|c|c|c|}
\hline \begin{tabular}{|l} 
IoT \\
platform
\end{tabular} & \begin{tabular}{|l|} 
Common \\
communicatio \\
n protocols \\
HTTP, CoAP, \\
MQTT, etc.) \\
\end{tabular} & $\begin{array}{l}\text { Security } \\
\text { (authentication, } \\
\text { authorizatioand } \\
\text { encryption) }\end{array}$ & \begin{tabular}{|l} 
Data \\
Format \\
Supported
\end{tabular} & $\begin{array}{l}\text { (platforms } \\
\text { have } \\
\text { strong } \\
\text { analytic } \\
\text { capabilitie } \\
\end{array}$ & \begin{tabular}{|l|} 
Programmi \\
ng \\
Languages \\
Support \\
\end{tabular} \\
\hline $\begin{array}{l}\text { Amazon } \\
\text { Web } \\
\text { Services } \\
\text { (AWS) } \\
\end{array}$ & $\begin{array}{l}\text { HTTP, MQTT, } \\
\text { WebSocket }\end{array}$ & \begin{tabular}{|l} 
Encryption \\
Authentication \\
Authorization
\end{tabular} & JSON & Yes & \begin{tabular}{|l|} 
C, Java, \\
NodeJS, \\
Javascript, \\
Python, \\
Arduino, \\
Android, \\
iOS \\
\end{tabular} \\
\hline $\begin{array}{l}\text { Google IoT } \\
\text { Platform }\end{array}$ & HTTP, MQTT & Authen & JSON & Yes & $\begin{array}{l}\text { Java, .NET, } \\
\text { Node.js, } \\
\text { php, Python, } \\
\text { Ruby }\end{array}$ \\
\hline $\begin{array}{l}\text { IBM } \\
\text { Watson }\end{array}$ & $\begin{array}{l}\text { HTTP, MQTT, } \\
\text { REST API }\end{array}$ & $\begin{array}{l}\text { Authentication } \\
\text { Authorization }\end{array}$ & JSON, CSV & Yes & \begin{tabular}{|l|} 
C\#, C, \\
Python, \\
Java, \\
NodeJS \\
\end{tabular} \\
\hline $\begin{array}{l}\text { Microsoft } \\
\text { Azure }\end{array}$ & $\begin{array}{l}\text { HTTPS, } \\
\text { MQTT, } \\
\text { WebSocket, } \\
\text { AMQP }\end{array}$ & $\begin{array}{l}\text { Encryption } \\
\text { Authentication } \\
\text { Authorization }\end{array}$ & JSON & Yes & $\begin{array}{l}\text { C, .NET, } \\
\text { Java, } \\
\text { NodeJS, } \\
\text { Ruby, } \\
\text { Android, } \\
\text { iOS }\end{array}$ \\
\hline Kaa & MQTT & Encr & $\begin{array}{l}\text { REST API, } \\
\text { JSON }\end{array}$ & $\begin{array}{lr}\text { Yes, } & \text { but } \\
\text { not } & \text { real } \\
\text { time } & \\
\text { analytics }\end{array}$ & $\begin{array}{l}\text { Java, C, } \\
\mathrm{C}++\end{array}$ \\
\hline Oracle IoT & $\begin{array}{l}\text { HTTP, MQTT, } \\
\text { CoAP, } \\
\text { WebSocket, } \\
\text { XMPP, and } \\
\text { AMQP }\end{array}$ & $\begin{array}{l}\text { Authentication } \\
\text { Authorization }\end{array}$ & $\begin{array}{l}\text { REST API, } \\
\text { CSV }\end{array}$ & $\begin{array}{l}\text { Yes, but } \\
\text { not } \\
\text { machine } \\
\text { learning }\end{array}$ & $\begin{array}{l}\text { C, Java, } \\
\text { Javascript, } \\
\text { Android, } \\
\text { iOS }\end{array}$ \\
\hline
\end{tabular}

\section{Challenges, Recommendations And Future DIRECTIONS OF IOT BASED IN-HOME HEALTH MONITORING SYSTEMS}

As the IoT technologies are becoming affordable and increasingly accepted by patients and healthcare workers, innovative applications are blooming and continue to be fueled by venture capitals and research funds from both private sectors and government. The prospect of IoT for eHealth is promising, new functions are being added into products and services with technological advances in sensor abilities in hardware, ubiquitous communication, and analytics supports of cloud/fog/big data computing. eHealth is listed as a priority in the European Digital Agenda in Horizon 2020 [34]. A saving of almost 100 billion Euro is possible in healthcare expenses with the aid of eHealth and mHealth. It is expected that the market value of all the wearable devices that measure fitness and wellness worldwide will be over $\$ 12$ billion USD by 2022 [35]. Recently a buffet of eHealth applications tapping on the power of IoT has emerged, ranging from ambient assisted living for illness prevention to paramedic emergency services for rescue.

A fully integrated in-home health monitoring system, with optimized workflows at the hospitals, is arriving soon in the future. Since chronic disease treatment and rehabilitation is not a once-off event, a full array of IoT sensors should be in place to facilitate bodily and environmental monitoring as a holistic approach. IoT plays an important role in enabling data links from multiple places for virtual consultation in future TeleHealth. Based on this, a doctor and patients' consultation can be equipped with not just lab results, but also with the history of data remotely measured while patients at home using some wearables and in-home remote patient monitoring applications. Using the available data and aided by decisionsupport systems that also have access to big data for other individuals, the doctor can make a better diagnosis and provide personalized treatment. Such a disruptive technology could have a transformative impact on global healthcare systems and drastically reduce healthcare costs and improve speed and accuracy for diagnoses [36].

The realization of this ambitious scenario and in general IoT based remote monitoring in the healthcare sector that requires data sharing and transfer between embedded devices is complex and gives rise to several challenges and open issues that need to be addressed in the future development of such systems.

IoT based in-home health remote monitoring is a network of systems, devices and sensors which are connected and share data. It is a platform which allows to manage the data and controls the devices remotely based on requirements. These requirements for IoT systems are measured by the quality of services (QoS) provision by these systems and the satisfaction of the end-users' requirements. This paper addresses the IoT challenges and open issues by mapping them to the IoT QoS and end-users' requirements. There is a range of quality factors that can be used to quantify QoS and many different Quality Models have been proposed to identify the QoS metrics in distributed systems. The ISO/IEC 25010 [37] quality model ISO/IEC (2010) is one of these models. It identifies QoS metrics as functional stability, performance, compatibility/interoperability, usability, reliability, security, maintainability/modularity/scalability and portability. The work in [38] mapped these metrics to IoT systems and identified reliability, performance, functional stability, security, compatibility/Interoperability and maintainability/scalability as QoS metrics in IoT architectures.

Based on this QoS list, this paper considers the following recommendation and future directions that need addressing in the future development of IoT based in-home health remote monitoring: 


\section{A. Performance, Functional stability and Reliability}

These QoS indicators are end-to-end based, applicable to all the sub-components of the IoT architecture starting from the individual's home to the healthcare cloud services. For the cloud services, it is to ensure that the cloud infrastructure can serve the maximum expected number of clients without dropping requests, and also to identify potential performance bottlenecks on the server-side. This will involve monitoring of the memory consumption to detect any problems with undesired memory leakage and badly designed caching of data. For the individual's home end this involves the performance, functional stability and reliability of the WBAN and WPAN networking concerning wireless interference from the coexistence of different short communications modalities (e.g. WiFi, ZigBee or BLE that employ the $2.4 \mathrm{GHz}$ ISM band). In particular, WiFi uses the highest transmission power compared to other technologies, operating at the same frequency band, resulting in a higher interference. Besides, the use of large packet sizes in WiFi technology affects the surrounding wireless devices' available bandwidth. Wireless interference can cause intermittent connectivity or unexpected disconnections, delays in connection and data transfer, slow network speeds, and poor signal strength. Many research efforts were conducted to study the cause and effect of the coexistence issues in WBAN (IEEE 802.15.6), WiFi, BLE and IEEE 802.15.4 [64]. Energy Consumption Management is another performance metric that can lead to degradation in performance/reliability and functional stability. For example, at the individual home end continuous monitoring scenarios mean wearables need to be active for a long amount of time and these can be hours to days, it depends on the clinical scenario. This means draining the battery that will need re-charging and hence disconnection for the continuous monitoring process. A low battery charge causes symptoms similar to that caused by wireless interference. Performance/reliability and functional stability are vital QoS indicators. Systems with low performance/reliability and functional stability lead to low adoptions and acceptance by end-users of such systems [39]. Developers, testers, evaluators and device manufacturers need to address and solve such indicators. This could eventually lead to higher adoption of such systems and a scale-up of such services in the healthcare sector that as reported in the literature can lead to a better quality of life and low cost.

\section{B. Security, Privacy. Ethics and Law}

Addressing security and privacy issues in IoT systems design and development is vital to develop trust in using IoT based systems in the healthcare domain. Security mechanisms must be embedded (privacy by design [40]) at every layer and every component of IoT architecture to prevent security threats and attacks and preserve privacy. There are many commercial and personal devices being created without due diligence to ensure these security and privacy aspects [41]. Developers need to ensure that the "things" that make up the IoT and the systems to which they connect to are secure; that the sensors, devices, gateways, IoT services can be trusted by the users and their identity, safety and privacy protected and maintained. In many in-home health IoT based remote monitoring applications, the developers rely on the embedded security mechanisms' (e.g. encryption) in the manufactured hardware and software components of the sensors and the transceivers of the wireless communications. Mostly, the developers of the data acquisition applications of such sensors and devices base their design of such applications on the principles of privacy by design and employ all the possible security mechanisms in terms of authorization and authentications, anonymization and encryption both when data stored and mobile. Many such applications usually are connected to existing healthcare information services that have their own security mechanisms and privacy policies applied, but may not be up-to-date with the latest security standards and mechanisms.

Privacy protection solutions need to help users decide who should legitimately access and alter information. Users of IoT must have confidence that their data collection, storage and usage is being done in a manner that benefits them and does no harm to their privacy. In general several guidelines and regulations already addressed privacy in developing IoT applications, such as the Health Insurance Portability and Accountability Act (HIPAA) [42], and the EU General Data Protection Regulation (GDPR) [40]. However, there is a need to consider the secondary use of the collected data using inhome IoT remote monitoring. The patients involved in using such systems may consent for their data to be used for the primary purpose of the in-home health remote monitoring and not the future/secondary use, e.g. building algorithms using the collected data (Big data analytics) [44].

With the development of IoT, several use cases are supported but it has also caused an increase to critical ethical and legal violations [48]. IoT based applications require an appreciation of both the ethico-legal milieu and the socio-political landscape. The requirements of the social license for research are: Reciprocity, non-exploitation and Service of the public good [43]. For these criteria to be satisfied, there is a need in including the public in the dialogues that concern their collected data. Research that improves NHS care needs to satisfy the requirements of non-exploitation and service of the public good. However, misuse of data being collected via (e.g. such IoT applications), without consultation, infringes these requirements [44]. It has been noticed that one of the issues in the low adoption of IoT applications among end-users is the lack of trust in IoT devices concerning data protection, privacy and safety. Therefore, this issue must be considered to improve trust among people using IoT devices and systems.

\section{Interoperability}

IoT interoperability can be defined as "the ability of two systems to communicate and share services with each other" [45]. IoT interoperability can be classified as device interoperability, networking interoperability, syntactic interoperability, semantic interoperability, and platform interoperability [46]. Interoperability plays a major role in IoT development and particularly in the healthcare domain. IoT based in-home health remote monitoring incorporates heterogeneous IoT sensors, devices, applications and services, 
which involves high amounts of data exchange in heterogeneous formats. Hence, interoperability should be considered by IoT developers and medical device manufacturers when developing IoT based remote monitoring systems. Many medical devices e.g. Glucose meter, blood pressure devices are based on the ISO/IEEE 11073 Personal Health Data (PHD) interoperability standards in their data communication that direct the developers of IoT application at the data acquisition side to use the same. However, other IoT devices are developed based on oneM2M communication protocol. Hence it is necessary to have some mechanisms that make PHD and oneM2M being able to talk to each other within the same IoT system [47].

Although many IoT standards and platforms have been developed and helped in advancing IoT interoperability issues, there are still some open research challenges to be solved [45]:

- To focus on cross-layer cross-platform interoperability than just the sensor/device and network layer interoperability using semantic web technologies and internetworking APIs.

- The interoperability should be made possible irrespective of the underlying technologies (e.g. non IoT devices).

- Interoperability testing needs to be automated instead of the current complex process of interoperability testing that includes all the stakeholders of technology providers, developers and end-users that may affect proper interoperability testing and in many cases may affect security mechanisms testing.

- Adopting public RESTAPI to access the resources rather than custom RESTAPI which makes mashing up of data across platform difficult. Most IoT platforms provide public RESTAPI, but still, some IoT platforms use custom RESTAPI.

- Development of unified IoT gateway platform to consider many standardized communications protocols where the device manufacturers may select the appropriate protocols (e.g. CoAP for constrained devices). Or they maybe free interoperability gateway solutions for facilitating the device to device (D2D) communication.

Interoperability challenges get more difficult to handle with the increased complexity of interconnected IoT systems in terms of technologies, integrated services, information and Open access, human factors and cross-border governance frameworks [66]. ISO/IEC 21823-1:2019(E) [67] is one of the current Interoperability for IoT systems framework standards to address these difficulties and provides a common understanding of interoperability in general IoT systems and the various elements within them.

\section{Scalability}

Scalability is another important aspect of IoT future direction in in-home health remote monitoring. It means the possibilities of adding new, services, sensors, devices, and applications without affecting the overall performance of the system. Scalability means developing IoT platforms that support a large number of sensors, devices, serves with different storage, processing, communications and bandwidth requirements [68][69]. Both the middleware layer and cloud services are great examples of scalability. They provide sufficient support to scale up the IoT system when adding new sensors, devices and service requirements. This direction has led to a new research paradigm in developing a unified IoT framework that satisfies global requirements.

\section{CONCLUSION}

IoT technologies have matured since its conception a decade ago, with increasingly successful implementations at smart city and smart home projects around the world. eHealth, however, has its root from the era of modern digitization movements since decades ago, from revamping electronic health records to telemedicine provision. By tapping on the ubiquitous computing power and sensing ability, IoT fueled eHealth applications to improve and foster better diagnostics, consultation, drug administration, and preventive advice. It is found that IoT plays the main role in gathering and disseminating information either to the users themselves as health status feedback, within the healthcare organization, or between the patients and remote doctors for fuller information about the users' current and past health conditions and living environment. It is foreseen that more initiatives along this direction will emerge, catching up in time with the lucrative funding opportunities by many countries worldwide. It is also evident that both investments in the eHealth industry and startups on IoT and eHealth companies snowballed in recent years.

New ideas are exploited, existing medical pathways and processes are enhanced by IoT technologies which create benefits in the value-chain. In the near future, conceptual prototypes would be developed and fuse into daily eHealth operations which eventually become an integral part of our life in healthcare. Despite all the advantages of healthcare systems, several open issues continue to exist, (performance, reliability, functional stability), (security, privacy, legal and ethics), (compatibility, interoperability) and (maintainability, scalability) among others.

$\mathrm{AI}$ and Big data will play an important role in advancing IoT in-home health remote monitoring. Linking such systems with existing data related to other individuals can provide a more personalized treatment plan for individuals. As an example, as more people with diabetes become connected via the use of apps and automated glucose sensors that measure interstitial glucose continuously, the amount of data on glycaemic control will expand massively. The clinician will not only be able to assess long-term control via $\mathrm{HbAlc}$, but also glucose levels minute-to-minute. AI will power apps that provide individualized guidance for people with chronic conditions e.g. adjustments of their treatment regimen and lifestyle recommendations.

In addition, another important challenge is the gap between the IoT technology community and the medical community. The technology of IoT based healthcare monitoring is being progressed quickly today. However, it is not widely used in the medical community such as hospitals; the medical communities still hesitate to adopt the remote monitoring technology due to some reasons. For example, how will an insurance company pay the remote diagnosis charge (e.g., in USA)? We want to note that IoT based remote monitoring is not the replacement of 
traditional face to face diagnosis, and is more like an assistive technology that can enhance the current medical approaches.

As the evolution of society, diseases and technologies continues, new mHealth scenarios and applications will appear and become a reality, these identified open issues will be ongoing. The research communities need to continue reporting on the benefits, challenges and lessons learned of IoT in-home health monitoring systems deployment so that new system development can learn from.

This paper presents the needs, the technologies advances, the challenges, recommendations and future directions of IoT based in-home health monitoring systems.

\section{REFERENCES}

[1] WHO, mhealth: New Horizon for Health Through Mobile Technologies (Global Observatory for e-Health Services), vol. 3. Geneva, Switzerland: WHO, 2011.

[2] WHO, WHO guideline recommendations on digital interventions for health system, 2019, ISBN: 978-92-4-155050-5 [Online]. Available: https://www.who.int/reproductivehealth/publications/digitalinterventions-health-system-strengthening/en/.

[3] R. S. H. Istepanian, E. Jovanov, and Y. T. Zhang, "Guest editorial introduction to the special section on m-health: Beyond seamless mobility for global wireless healthcare connectivity," IEEE Trans. Inf. Technol. Biomed., vol. 8, no. 4, pp. 405-412, Dec 2004.

[4] WHO, "Key facts: Mental health of older adults," 2017. [Online]. Available: https://www.who.int/news-room/fact-sheets/detail/mentalhealth-of-older-adults

[5] Market Watch, 'mHealth Market 2019: Industry Analysis, Size, Share, Growth by 2025 - Global Market insights', 2019. [Online]. Available: https://www.marketwatch.com/press-release/mhealth-market-2019industry-analysis-size-share-growth-by-2025---global-market-insights2019-07-29.

[6] NHS Long Term Plan, 2019, Available: https://www.longtermplan.nhs.uk/.

[7] D. Miorandi, S. Sicari, F. De Pellegrini, I. Chlamtac, 'Internet of things: Vision, applications and research challenges' Ad Hoc Networks', Volume 10, Issue 7, September 2012, Pages 1497-1516.

[8] A. Froytlog et al., "Ultra-Low Power Wake-up Radio for 5G IoT," in IEEE Communications Magazine, vol. 57, no. 3, pp. 111-117, March 2019.

[9] K. Guk, G. Han, J. Lim, K. Jeong, T. Kang, E. K. Lim \& J. Jung, (2019). Evolution of Wearable Devices with Real-Time Disease Monitoring for Personalized Healthcare. Nanomaterials (Basel, Switzerland), 9(6), 813. doi:10.3390/nano9060813

[10] A. Lee \& K. Lee, 'The Internet of Things (IoT): Applications, investments, and challenges for enterprises', Business Horizons, Volume 58, Issue 4, July-August 2015, Pages 431-440.

[11] S. Kitsiou, G. Paré, M. Jaana, B. Gerber, 'Effectiveness of mHealth interventions for patients with diabetes: An overview of systematic reviews'. PLoS One. 2017;12(3):e0173160. Published 2017 Mar 1. doi:10.1371/journal.pone.0173160].

[12] 'The Rise of Remote Patient Monitoring', May 2019, [Online], Available: https://thejournalofmhealth.com/the-rise-of-remote-patient-monitoring/ .

[13] A. Bui, G. Fonarow, 'Home monitoring for heart failure management' . Journal of the American College of Cardiology 2012;59(2):97-104. doi:10.1016/j.jacc.2011.09.044.

[14] I. Chouvarda, N. Philip, P. Natsiavas, et. al, 'WELCOME - Innovative Integrated Care platform using Wearable Sensing and Smart Cloud Computing for COPD patients with Comorbidities', IEEE Engineering in medicine and biology society. August 2014. S. Turner, "New directions in communications," IEEE J. Sel. Areas Commun., vol. 13, no. 1, pp. 1123, Jan. 1995.

[15] J. Wacker et al., "Electrical and mechanical design of a vest measuring a large set of physiological signals," 2014 4th International Conference on Wireless Mobile Communication and Healthcare - Transforming Healthcare Through Innovations in Mobile and Wireless Technologies (MOBIHEALTH), Athens, 2014, pp. 47-50.

[16] Postcapes . 2017. "IoT Standards and Protocols," [Online]. Available: https://www.postscapes.com/internet-of-things-protocols/ ..

[17] A. Saboor, R. Ahmad, W.Ahmed, A. K. Kiani, Y. L. Moullec, M. M. Alam, 'On Research Challenges in Hybrid Medium Access Control Protocols for IEEE 802.15.6 WBANs',. IEEE Sens. J. 2018, 19, 1.

[18] N. Philip et al., "Design of a RESTful middleware to enable a web of medical things," 2014 4th International Conference on Wireless Mobile Communication and Healthcare - Transforming Healthcare Through Innovations in Mobile and Wireless Technologies (MOBIHEALTH), Athens, 2014, pp. 361-364.

[19] F. C. Delicato, P. F. Pires, and J. Katz, "Middleware Solutions for the Internet of Things," 2013. Springer Publishing Company.

[20] S. Bandyopadhyay, M. Sengupta, S. Maiti, and S. Dutta, "Role of Middleware for Internet of Things: A Study," Int. J. Comput. Sci. Eng. Surv., vol. 2, no. 3, pp. 94-105, 2011..

[21] C. Perera, P. P. Jayaraman, and P. Christen, "MOSDEN : An Internet of Things Middleware for Resource Constrained Mobile Devices," in 47th Hawaii International Conference on System Sciences (HICSS). 2014.

[22] V. Scuturici, J. Petit, and U. De Lyon, "UbiWare : Web-based Dynamic Data \& Service Management Platform for AmI," pp. 5-6.

[23] "HYDRA." [Online]. Available: http://www.hydramiddleware.eu.

[24] "REACTION." [Online]. Available: http://www.reaction-project.eu.

[25] "inCASA." [Online]. Available: http://www.incasa-project.eu.

[26] "ebbits." [Online]. Available: http://www.ebbits-project.eu.

[27] "MASSIF." [Online]. Available: http://www.massif-project.eu

[28] "SEEMPubS." [Online]. Available: http://seempubs.polito.it.

[29] "SEAM4US." [Online]. Available: http://seam4us.eu/.

[30] A. J. Jara, M. A. Zamora-izquierdo, and A. F. Skarmeta, "Interconnection Framework for mHealth and Remote Monitoring Based on the Internet of Things," IEEE JSAC, vol. 31, no. 9, pp. 47-65, 2013

[31] "OpenIoT." [Online]. Available: http://openiot.eu/.

[32] M\&S Consulting . 2017. "Industrial Internet of Things Platform Comparison,"[Online]. Available: https://www.mandsconsulting.com/industrial-iot-platform-comparison.

[33] D. Sobnath, N. Philip, R. Kayyali, et. al, "Features of a COPD Patient Support Mobile Application: Review of the Literature and Analysis of Current Applications." Journal of Medical Internet Research MHealth and UHealth 5 (2). https://doi.org/10.2196/mhealth.4951

[34] Shaping Europe's digital future, POLICY, Research and Innovation in eHealth, [Online]. Available: https://ec.europa.eu/digital-singlemarket/en/research-and-innovation-ehealth.

[35] Wearable Fitness Technology Market Report, 2016, [Online], Available: https://www.marketsandmarkets.com/Market-Reports/wearable-fitnesstechnology-market-139869705.html.

[36] M. Hassanalieragh et al., "Health Monitoring and Management Using Internet-of-Things (IoT) Sensing with Cloud-Based Processing: Opportunities and Challenges," 2015 IEEE International Conference on Services Computing, New York, NY, 2015, pp. 285-292.

[37] ISO/IEC 25010 - Systems and software engineering - Systems and software Quality Requirements and Evaluation (SQuaRE) - System and software quality models Technical Report (2010)

[38] G. White, V. Nallur S.Clarke. 'Quality of service approaches in IoT: a systematic mapping'. J Syst Softw. 2017;132:186-203.

[39] E. Kaimakamis et al., 'Experience of Using the WELCOME Remote Monitoring System on Patients with COPD and Comorbidities', Precision Medicine Powered by pHealth and Connected Health. ICBHI 2017. IFMBE Proceedings, vol 66. Springer, Singapore

[40] A guide to GDPR data privacy requirements, https://gdpr.eu/dataprivacy/.

[41] F. D. Hudson, P. A. Laplante and B. Amaba, "Enabling Trust and Security: TIPPSS for IoT," in IT Professional, vol. 20, no. 2, pp. 15-18, Mar./Apr. 2018. doi: 10.1109/MITP.2018.021921646

[42] The HIPAA Privacy Rule. [Online]. Available: https://www.hhs.gov/hipaa/for-professionals/privacy/index.html

[43] P. Carter, GT.Laurie, M. Dixon-Woods, "The social licence for research: why care.data ran into trouble," in J Med Ethics. 2015;41(5):404-9.

[44] J.M.M. Rumbold, et al., 'Big data and diabetes: the applications of big data for diabetes care now and in the future," in Diabetic Medicine., 2019. ISSN 0742-3071

[45] J. Kiljander et al., "Semantic Interoperability Architecture for Pervasive Computing and Internet of Things," in IEEE Access, vol. 2, pp. 856-873, 2014.

[46] K. Park, J. Park, J. Lee, "An IoT System for Remote Monitoring of Patients at Home". Appl. Sci. 2017, 7, 260.

[47] C. Pereira, A. Aguiar, "Towards efficient mobile M2M communications: survey and open challenges," in Sensors. 2014;14(10):19582-608 
[48] S.G. Tzafestad, "Ethics and law in the internet of things world," in Smart Cities. 2018;1(1):98-120.

[49] C. Seeger, K. Van Laerhoven and A. Buchmann, "MyHealthAssistant: An Event-driven Middleware for Multiple Medical Applications on a Smartphone-Mediated Body Sensor Network," in IEEE Journal of Biomedical and Health Informatics, vol. 19, no. 2, pp. 752-760, March 2015.

[50] R. Zgheib, et al., "Semantic Middleware Architectures for IoT Healthcare Applications, Enhanced Living Environments. Lecture Notes in Computer Science, 2019, vol 11369. Springer, Cham.

[51] M. A. A. da Cruz, Joel J. P. C. Rodrigues, et al., , 'Performance evaluation of IoT middleware', Journal of Network and Computer Applications, Volume 109, 1 May 2018, Pages 53-65.

[52] T. Chomutare, L. Fernandez-Luque, et al., " Features of Mobile Diabetes Applications: Review of the Literature and Analysis of Current Applications Compared Against Evidence-Based Guidelines”, J Med Internet Res 2011;13(3).

[53] R. Goffredo, D. Accoto, M. Santonico, G. Pennazza and E. Guglielmelli, "A smart pill for drug delivery with sensing capabilities", 37th Annual International Conference of the IEEE Engineering in Medicine and Biology Society (EMBC), Milan, 2015, pp. 1361-1364.

[54] Ra et al., "AsthmaGuide: an asthma monitoring and advice ecosystem," 2016 IEEE Wireless Health (WH), Bethesda, MD, 2016, pp. $1-8$.

[55] T. Antonio, et al., "Tricoli, Noushin Nasiri, Sayan De, Wearable and Miniaturized Sensor Technologies for Personalized and Preventive Medicine," in Advanced Functional Materials, February 2017, DOI: 10.1002/adfm.201605271, pp. 1- 19

[56] M. Marie-Valérie and H. Edouard, "Evaluation on Phantoms of the Feasibility of a Smart Bra to Detect Breast Cancer in Young Adults," in Sensors. 2019 Dec; 19(24): 5491.

[57] M. G. Eshghi, Z. Fruhideh, V. M. Alviri and M. Modarresi Asem, "Electrochemical Biosensors for Cancer Detection Using Different Biomarkers," 2019 IEEE 9th Annual Computing and Communication Workshop and Conference (CCWC), Las Vegas, NV, USA, 2019, pp. 0989-0996..

[58] C. Ornetzeder, F. Reiterer, M. B. Christensen, K. Nørgaard, G. Freckmann and L. del Re, "Feasibility of Fully Closed Loop Insulin Delivery in Type 2 Diabetes," 2019 IEEE Conference on Control Technology and Applications (CCTA), Hong Kong, China, 2019, pp. 906913..

[59] P. Sever and M. Niculescu, "Portable Optical Coagulation Analyzer Based on Real-Time Image Processing Algorithm," 2019 11th International Symposium on Advanced Topics in Electrical Engineering (ATEE), Bucharest, Romania, 2019, pp. 1-6.

[60] T. Aledavood, et al., "Smartphone-Based Tracking of Sleep in Depression, Anxiety, and Psychotic Disorders," Curr Psychiatry Rep 21, 49 (2019). https://doi.org/10.1007/s11920-019-1043-y

[61] H. S. Gauvin, et al., "Verbal monitoring in Parkinson's disease: A comparison between internal and external monitoring," PLOS ONE (2017) 12(8): e0182159. https://doi.org/10.1371/journal.pone.0182159.

[62] R. José J. R. Barata, Roberto Munoz, Rafael D. de Carvalho Silva, Joel J. P. C. Rodrigues, and Victor Hugo C. de Albuquerque, "Internet of Things based on Electronic and Mobile Health Systems for Blood Glucose Continuous Monitoring and Management", IEEE Access Journal, Vol. 7, N. 1, December 2019, pp. 175116-175125, DOI: 10.1109/ACCESS.2019.2956745.

[63] F. Bonomi, R. Milito, J. Zhu, S. Addepalli, "Fog Computing and Its Role in the Internet of Things," Proceedings of the first edition of the MCC workshop on Mobile cloud computing, August 2012, Helsinki, Finland.

[64] $\mathrm{H}$. Hayajneh, et al. A survey of wireless technologies coexistence in WBAN: Analysis and open research issues, Wireless Networks 20(8): Nov 2014.

[65] M. A. A. da Cruz, Joel J. P. C. Rodrigues, Jalal Al-Muhtadi, Valery Korotaev, Victor Hugo C. Albuquerque, "A Reference Model for Internet of Things Middleware", in IEEE Internet of Things Journal, IEEE, ISSN: 2327-4662, Vol. 5, No. 2, April 2018, pp. 871-883, DOI: 10.1109/JIOT.2018.2796561

[66] The New European Interoperability Framework (EIF). [Online]. Available: https://ec.europa.eu/isa2/eif en .

[67] ISO/IEC 21823-1:2019 - Interoperability for IoT systems - Part 1: Framework. [Online]. Available: https://www.iso.org/standard/71885.html.
[68] S. Kumar, et al., "Internet of Things is a revolutionary approach for future technology enhancement: a review," in J Big Data 6, 111 (2019). https://doi.org/10.1186/s40537-019-0268-2

[69] M. Imran, S. Jabbar, N. Chilamkurti, J. J. P. C. Rodrigues, "Enabling technologies for Social Internet of Things", in Future Generation Computer Systems, Elsevier, ISSN: 0167-739X, Vol. 92, March 2019, pp. 715-717, DOI: 10.1016/j.future.2018.11.018.

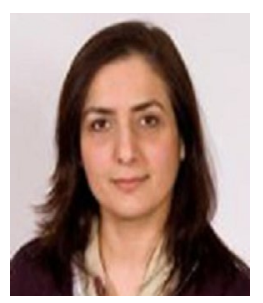

Dr Nada Y. Philip is an Associate Professor in the field of Mobile health (mHealth) at Kingston University London. She is the founder of the research group Digital Media for Health in 2012. She obtained her $\mathrm{PhD}$ in mHealth with the thesis title 'Medical Quality of Service for Optimised Ultrasound Streaming in Wireless Robotic Tele-ultrasonography System' from Kingston University, UK in 2008. Her research interests are mainly in the advancement of Data and Multimedia Communication, Networking and Information Technology for healthcare applications. She is the PI and Co-PI of many national and international mHealth projects in the areas of personalized health for Diabetes, Cancer and COPD conditions, $5 \mathrm{G}$ health, wearables and cloud computing, IoT, AI and Big data analytics for health, social robotics for health, end to end QoS and QoE in medical video streaming. She is the author and co-author of more than 80 journals, peer reviewed conferences and book chapters. She is a member of the editorials and the review panels for many journals including IEEE-IoT, JSAC and WCMC. She is the editor of the IEEE ehealth TC newsletter. She is a reviewer on both the MRC and the NIHR research bodies. She is a fellow of the Higher Education Academy, Senior Member of the IEEE, IEEE communication society and the IEEE Engineering in Medicine and Biology Society (EMBS).

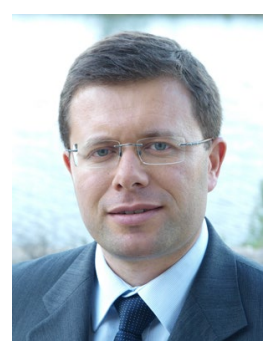

Joel J. P. C. Rodrigues [S'01, M'06, SM'06, F'20] is a professor at the Federal University of Piauí, Brazil; senior researcher at the Instituto de Telecomunicações, Portugal; and collaborator of the Post-Graduation Program on Teleinformatics Engineering at the Federal University of Ceará (UFC), Brazil. Prof. Rodrigues is the leader of the Next Generation Networks and Applications (NetGNA) research group $(\mathrm{CNPq})$, an IEEE Distinguished Lecturer, Member Representative of the IEEE Communications Society on the IEEE Biometrics Council, and the President of the scientific council at ParkUrbis - Covilhã Science and Technology Park. He was Director for Conference Development - IEEE ComSoc Board of Governors [20182019], Technical Activities Committee Chair of the IEEE ComSoc Latin America Region Board [2018-2019], a PastChair of the IEEE ComSoc Technical Committee on eHealth, a Past-chair of the IEEE ComSoc Technical Committee on Communications Software, a Steering Committee member of the IEEE Life Sciences Technical Community and Publications 
co-Chair [2014-2017]. He is the editor-in-chief of the International Journal on E-Health and Medical Communications and editorial board member of several highreputed journals. He has been general chair and TPC Chair of many international conferences, including IEEE ICC, IEEE GLOBECOM, IEEE HEALTHCOM, and IEEE LatinCom. He has authored or coauthored over 900 papers in refereed international journals and conferences, 3 books, 2 patents, and 1 ITU-T Recommendation. He had been awarded several Outstanding Leadership and Outstanding Service Awards by IEEE Communications Society and several best papers awards. Prof. Rodrigues is a member of the Internet Society, a senior member ACM, and Fellow of IEEE.

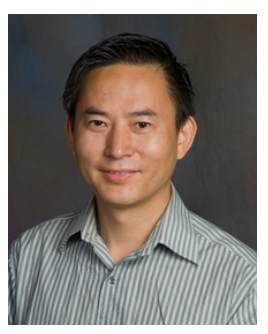

Prof. Honggang Wang's research interests include Internet of Things, Wireless Body Area Networks (BAN), Multimedia Communication and Applications, Wireless Health, Cyber and Multimedia Security, Wireless Networks and Cyberphysical System, Connected Vehicle and BIG DATA in mHealth. He has published more than 200 papers in his research areas, and most of them appeared in prestigious IEEE journals and conferences. As an author/co-author, he has received several best conference paper awards from conferences such as IEEE GLOBECOM and IEEE WCNC. He has also been serving as the Editor in Chief (EiC) of IEEE Internet of Things journal (SCI impact factor: 9.5), the technical Editor of IEEE Transactions on Vehicular Technology, associate editors for IEEE Transactions on Multimedia, IEEE Transactions on Big Data, and IEEE Network Magazine. He currently serves as the Chair (20202021) of IEEE eHealth Technical Committee. His research is supported by NSF (6 grants), DoT, NIH, UMass President office, and UMass Healey Grant (total over 3 M dollars). He received UMass Dartmouth Sponsored Research Recognition Award in 2015. His research is reported by media such as USA ABC 6 TV, Standard Times Newspaper. $\mathrm{He}$ is an IEEE distinguished lecturer.

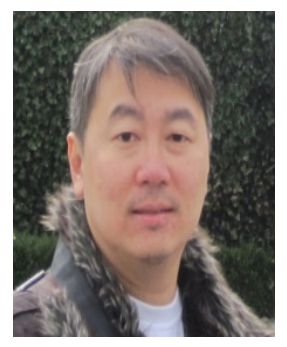

Simon James Fong is an Associate Professor at the Computer and Information Science Department of the University of Macau, also an Adjunct Professor at Faculty of Informatics, Durban University of Technology, South Africa, at ZIAT of Chinese Academy of Science, and at Department of Computer Science, Xi'an Polytechnic University, Xi'an, China. He is a co-founder of the Data Analytics and Collaborative Computing Research Group in the University of Macau. Prior to his academic career, Simon took up various managerial and technical posts, such as systems engineer, IT consultant and ecommerce director in Australia and Asia. Simon has published over 450 international conference and peer-reviewed journal papers, mostly in the areas of data mining, big data analytics, meta-heuristics optimization algorithms and their applications. He serves on the editorial boards of the Journal of Network and
Computer Applications of Elsevier, IEEE IT Professional Magazine, and various special issues of SCIE-indexed journals. Simon is also an active researcher with leading positions such as Vice-chair of IEEE Vice-director of International Consortium for Optimization and Modelling in Science and Industry (iCOMSI).

Dr. Jia Chen is an Offering Leader of Blockchain solutions for Healthcare and Life Sciences at IBM's Innovation and Solution Incubation team. She serves on the IBM Academy of Technology Leadership team. She previously led technical strategy at IBM Watson Health Innovation, with a focus on data and AI. Prior to that, Dr. Chen was the global leader of Watson Experience Centers at IBM, responsible for Watson AI client experiences across all Watson group. She held leadership positions for Innovation and client engagement at IBM Corporate Headquarters as well as emerging markets. She was formerly the Director of Health Solutions for Smarter Cities at IBM, and the Director of Technical Sales \& Innovation for IBM's Growth Market Units. She led the identification, structuring and execution of first of a kind technology and business initiatives that provide innovative and sustainable differentiation for IBM's clients. Dr. Chen received her Ph.D. in Physics from Yale University. She was named as one of the top 35 technology innovators under the age of 35 worldwide by MIT's Technology Review in 2005, the Best Researcher of the Year by Small Times magazine in 2006 and one of the top 26 tech women innovators at IBM in 2015. She serves on the Yale Graduate School Alumni Association Board. 